\title{
Perfil dos cuidadores e as dificuldades enfrentadas no cuidado ao idoso, em Ananindeua, PA
}

\author{
Profile of caregivers and difficulties in elderly care in the city of Ananindeua, State of Para, Brazil
}

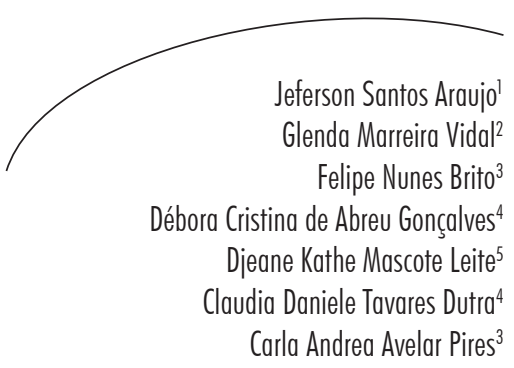

Resumo

Objetivo: Descrever o perfil dos cuidadores de idoso, bem como sua importância e principais dificuldades no ato de cuidar do idoso. Metodologia: Trata-se de estudo transversal descritivo e exploratório realizado pela equipe PET Saúde, em uma Estratégia de Saúde da Família (ESF), no município de Ananindeua, Pará. A coleta de dados foi feita por meio de um questionário semiestruturado abordando aspectos sociodemográficos e questões referentes a importância e dificuldades encontradas pelos cuidadores de idosos. Resultados: Foram entrevistados 31 cuidadores, predominando sexo feminino, estado civil casado, com ensino médio completo, renda entre dois e três salários mínimos, tempo de exercício da função maior que 12 meses, em sua abrangência cuidadores informais e com vínculo de parentesco com o idoso. Os cuidadores reconheceram que é importante a presença de um cuidador e consideraram o idoso sob seu cuidado como saudável. Conclusão: A maioria dos cuidadores era do tipo informal, caracterizada por mulheres, com vínculo familiar, que destacaram a paciência e a falta de conhecimento como as principais dificuldades do cuidador. Os dados deste estudo se limitam a uma realidade local, necessitando de mais pesquisas para fomentar as particularidades e dificuldades enfrentadas pelos cuidadores.

Abstract

Objective: To describe the profile of caregivers of elderly, as well as their importance and main difficulties in caring for the elderly. Methodology: This was a descriptive, exploratory cross-sectional study conducted by the PET Saúde staff in a Family

\footnotetext{
Programa de Pós-graduação em Enfermagem Fundamental, Escola de Enfermagem de Ribeirão Preto. Universidade de São Paulo. Ribeirão Preto, SP, Brasil.

2 Programa de Pós-graduação em Nutrição. Universidade Federal de Santa Catarina. Florianópolis, SC, Brasil.

3 Faculdade de Medicina, Instituto de Ciências da Saúde. Universidade Federal do Pará. Belém, PA, Brasil.

4 Faculdade de Nutrição, Instituto de Ciências da Saúde. Universidade Federal do Pará. Belém, PA, Brasil

Programa de Educação pelo Trabalho para a Saúde (PET). Universidade Federal do Pará. Ananindeua, PA, Brasil.
}

Palavras-chave: Saúde Pública. Ensino. Assistência à Saúde. Cuidadores. Idoso. 
Health Strategy (FHS) in the city of Ananindeua, state of Pará, Brazil. Data was collected through a semistructured questionnaire addressing sociodemographic and issues concerning the importance and difficulties encountered by caregivers of the elderly. Results: We interviewed 31 caregivers, predominantly female, married state, with complete secondary education, income between two and three minimum wages, time of exercise of the function over 12 months, most of them informal caregivers and with family relationship with elderly. Caregivers acknowledged the importance of a caregiver and found the elderly under their care as healthy. Conclusion: Most caregivers were informal type, characterized by women with family ties, which emphasized patience and lack of knowledge as the main difficulties of the caregiver. Data from this study are limited to a local reality, requiring further research to foster the particularities and difficulties faced by caregivers.
Key words: Public Health. Teaching. Delivery of Health Care. Caregivers. Aged.

\section{INTRODUÇÃO}

Envelhecer é algo inexorável a todos os seres humanos, faz parte do ciclo vital e impossível de ser ultrapassado. ${ }^{1}$ Aos poucos, o corpo passa por um processo disfuncional e começa a reagir lento aos reflexos que outrora eram instantâneos. A mente começa a ter falhas, podendo chegar em muitos momentos até a esquecer sua real função; ${ }^{2}$ entretanto, também é no envelhecer que se descobrem muitas facetas da vida, valorizandose a transição do tempo e o passar de muitas trajetória vividas.

O envelhecer populacional é uma questão de saúde pública por ser um problema social do qual emergem gastos e investimentos governamentais. ${ }^{3}$ A frequência das doenças crônicas e a longevidade das pessoas têm causado o crescimento das taxas de idosos portadores de incapacidades. A prevenção das doenças e agravos não-transmissíveis, a assistência à saúde dos idosos dependentes e o suporte aos seus cuidadores familiares representam novos desafios para o sistema de saúde. ${ }^{4}$

O cuidador é a pessoa que presta cuidados à outra pessoa que esteja necessitando, por estar acamada, com limitações físicas ou mentais, com ou sem remuneração. ${ }^{5}$ É fundamental para a reabilitação e para o atendimento às necessidades cotidianas do idoso fragilizado, sobretudo no seguimento das orientações para a saúde, bem-estar, segurança, conforto e, ainda, no respeito e incentivo ao estimulo, à autonomia e independência. ${ }^{6}$

O cuidado prestado ao idoso exige dedicação exclusiva e quase sempre integral, que muitas vezes leva o cuidador à instalação de uma nova dinâmica de vida, baseada nas necessidades do ser cuidado. A busca pela promoção da autonomia e independência do idoso é tarefa árdua e desgastante para os cuidadores, pois estes passam a realizar tarefas que outrora eram de cunho pessoal e desenvolvido de maneira autônoma pelo idoso. ${ }^{7}$ O cuidador de idosos é aquele que convive diariamente com o idoso, prestando-lhe cuidados higiênicos, ajudando com a alimentação, administrando medicação e estimulando-o com as atividades reabilitadoras, interagindo, assim, com a equipe terapêutica. ${ }^{2-4}$

No Brasil, dados do censo do Instituto Brasileiro de Geografia e Estatística (IBGE) revelam que 14,5 milhões de indivíduos se encontram com mais de 60 anos de idade e necessitam diretamente de cuidadores. ${ }^{8}$ Hoje no país, entrentanto, pouco se conhece sobre os cuidadores de idosos, pois a maioria das literaturas foca nas estratégias de enfrentamento para os idosos dentro de patologias como câncer, acidente vascular cerebral e demências funcionais, ficando a figura de seus cuidadores em segundo plano. 
Dentro desse contexto, o presente estudo tem como objetivo descrever o perfil dos cuidadores de idoso (características sociodemográficas, vínculo com idoso, tipo de cuidador, tempo de serviço), bem como sua importância e suas principais dificuldades no ato de cuidar do idoso.

\section{METODOLOGIA}

$\mathrm{O}$ estudo foi transversal descritivo $\mathrm{e}$ exploratório. A população estudada constituiuse dos idosos e seus cuidadores atendidos pela equipe do PET-Saúde, na Estratégia de Saúde da Família (ESF) Seringal, no município de Ananindeua, no Estado do Pará, no período de setembro de 2010 a junho de 2011.

Para a coleta de dados, foi feito inicialmente o levantamento de quantos idosos eram cadastrados na ESF, buscando identificar quais os idosos que possuíam cuidadores.

Os critérios de inclusão foram: os cuidadores que se encontravam no domicílio no dia da visita e que prestavam assistência ao idoso há pelo menos um mês. Foram excluídos os participantes que não assinaram o Termo de Consentimento Livre e Esclarecido e os que não estavam presentes no dia da visita da ESF. Ressalta-se que os domicílios que foram selecionados eram distantes uns dos outros e de difícil acesso, por isso a equipe se planejoupara fazer uma única visita em cada residência.

Foram considerados cuidadores informais os familiares (filhos, mães, esposas, entre outros) que de alguma forma possuíam vínculo com o idoso, caracterizando-se por ser, acima de tudo, voluntários que se dispõem, sem formação profissional específica, a exercer esta função. ${ }^{2}$ Os cuidadores formais são os cuidadores que provêm os cuidados de saúde aos idosos, em função de sua profissão, e usam suas habilidades, a competência e a introspecção originadas em treinamentos específicos para cuidar do outro. ${ }^{3,4}$

A coleta de dados foi feita por meio de questionário estruturado destinado aos cuidadores, que constou de dados sociodemográficos (sexo, idade, estado civil, escolaridade e renda ), tipo de cuidador (formal ou informal), tipo de vínculo com o idoso, tempo que exercia na função, se o cuidador recebia alguma remuneração pela atividade, dificuldades encontradas no ato de cuidar do idoso, se ele considerava importante sua presença como cuidador e como ele classificava o estado de saúde do idoso que cuidava. Para a caracterização da amostra, utilizou-se a estatística descritiva. Para as variáveis contínuas, utilizaram-se medidas de tendência central e de dispersão conforme simetria; e para as variáveis categóricas, apresentou-se a frequência absoluta e relativa.

Os dados foram tabulados no programa Microsoft Excel 2007 e as análises foram efetuadas com o auxílio do Programa Stata 11.0.

A pesquisa envolveu seres humanos e foi realizada em conformidade com a Resolução n. 196/96 do Conselho Nacional de Saúde. O projeto foi aprovado pelo Comitê de Ética do Instituto de Ciências da Saúde da Universidade Federal do Pará, registro no 104/10-CEP-ICS/UFPA. Todos os participantes receberam informações sobre o estudo e assinaram o Termo de Consentimento Livre e Esclarecido.

\section{RESULTADOS}

A amostra foi composta por 31 cuidadores de idosos que se caracterizavam por ser mulheres, com idade média de 46,7 ( $\pm 12,7)$ anos, casadas, com ensino médio completo, renda entre dois e três salários mínimos e possuíam a função de cuidador há mais de 12 meses, conforme a tabela 1 .

A maioria era cuidador informal, em grande parte cônjuge ou filho. Apenas 3,2\% dos cuidadores recebiam remuneração pelo trabalho. A maior parte reconheceu ser importante a presença de um cuidador, e quase dois terços deles consideraram o idoso sob seu cuidado como saudável (tabela 1). 
Tabela 1 - Perfil dos cuidadores de idosos, ESF Seringal. Ananindeua, PA, 2010-2011.

\begin{tabular}{|c|c|c|c|}
\hline Variáveis & $\mathrm{n}$ & $\%$ & IC95\% \\
\hline \multicolumn{4}{|l|}{ Sexo } \\
\hline Masculino & 6 & 19,3 & 4,$6 ; 34,0$ \\
\hline Feminino & 25 & 80,7 & 65,$9 ; 95,4$ \\
\hline \multicolumn{4}{|l|}{ Estado civil } \\
\hline Solteiro(a) & 12 & 38,7 & 20,$5 ; 56,9$ \\
\hline Casado(a) & 18 & 58,1 & 40,$0 ; 76,5$ \\
\hline Divorciado(a) & 1 & 3,2 & 0,$0 ; 9,8$ \\
\hline \multicolumn{4}{|l|}{ Faixa etária (anos)* } \\
\hline$<20$ & 1 & 3,4 & 0,$0 ; 10,5$ \\
\hline 20 a 29 & 3 & 10,3 & 0,$0 ; 22,1$ \\
\hline 30 a 39 & 2 & 6,9 & 0,$0 ; 16,7$ \\
\hline 40 a 49 & 7 & 24,1 & 7,$5 ; 40,7$ \\
\hline 50 a 59 & 11 & 37,9 & 19,$1 ; 56,7$ \\
\hline$\geq 60$ & 5 & 17,2 & 2,$6 ; 31,9$ \\
\hline \multicolumn{4}{|l|}{ Escolaridade } \\
\hline Ensino fundamental incompleto & 5 & 16,1 & 2,$4 ; 29,8$ \\
\hline Ensino fundamental completo & 5 & 16,1 & 2,$4 ; 29,8$ \\
\hline Ensino médio completo & 17 & 54,9 & 36,$3 ; 73,4$ \\
\hline Ensino superior completo & 4 & 12,9 & 0,$4 ; 25,4$ \\
\hline \multicolumn{4}{|l|}{ Recebe remuneração } \\
\hline $\operatorname{Sim}$ & 1 & 3,2 & 0,$0 ; 9,8$ \\
\hline Não & 30 & 96,8 & 90,$2 ; 100,0$ \\
\hline \multicolumn{4}{|l|}{ Renda em salário mínimo } \\
\hline Nenhuma & 6 & 19,3 & 4,$6 ; 34,1$ \\
\hline Até 1 & 7 & 22,6 & 7,$0 ; 38,2$ \\
\hline 2 a 3 & 15 & 48,4 & 30,$0 ; 67,0$ \\
\hline$\geq 4$ & 3 & 9,7 & 0,$0 ; 20,7$ \\
\hline
\end{tabular}




\begin{tabular}{|c|c|c|c|}
\hline Variáveis & $\mathrm{n}$ & $\%$ & IC95\% \\
\hline \multicolumn{4}{|l|}{ Tipo de cuidador } \\
\hline Formal & 1 & 3,2 & 0,$0 ; 9,8$ \\
\hline Informal & 30 & 96,8 & 90,$2 ; 100,0$ \\
\hline \multicolumn{4}{|l|}{ Vínculo com o idoso } \\
\hline Esposa & 7 & 22,6 & 7,$0 ; 38,2$ \\
\hline Filho(a) & 13 & 41,9 & 23,$6 ; 60,3$ \\
\hline Sobrinho(a) & 4 & 12,9 & 0,$4 ; 25,4$ \\
\hline Genro ou nora & 2 & 6,5 & 0,$0 ; 15,6$ \\
\hline Neto(a) & 3 & 9,7 & 0,$0 ; 20,7$ \\
\hline Irmã & 1 & 3,2 & 0,$0 ; 9,8$ \\
\hline Nenhum & 1 & 3,2 & 0,$0 ; 9,8$ \\
\hline \multicolumn{4}{|c|}{ Tempo que exerce na função em meses* } \\
\hline 1 & 1 & 3,3 & 0,$0 ; 10,2$ \\
\hline 6 a 12 & 2 & 6,7 & 0,$0 ; 16,1$ \\
\hline$>12$ & 27 & 90,0 & 78,$6 ; 100,0$ \\
\hline \multicolumn{4}{|c|}{ Considera importante ter um cuidador* } \\
\hline $\operatorname{Sim}$ & 29 & 96,7 & 89,$8 ; 100,0$ \\
\hline Não & 1 & 3,3 & 0,$0 ; 10,1$ \\
\hline \multicolumn{4}{|l|}{ Considera o idoso saudável* } \\
\hline $\operatorname{Sim}$ & 23 & 76,7 & 60,$6 ; 92,7$ \\
\hline Não & 7 & 23,3 & 7,$3 ; 39,4$ \\
\hline
\end{tabular}

*Variáveis com dados ignorados.

ESF: Estratégia Saúde da Família; IC: Intervalo de Confiança. 
As principais dificuldades relatadas pelos cuidadores foram a falta de paciência, seguida da falta de formação para o desenvolvimento da função, conforme mostra a figura 1.

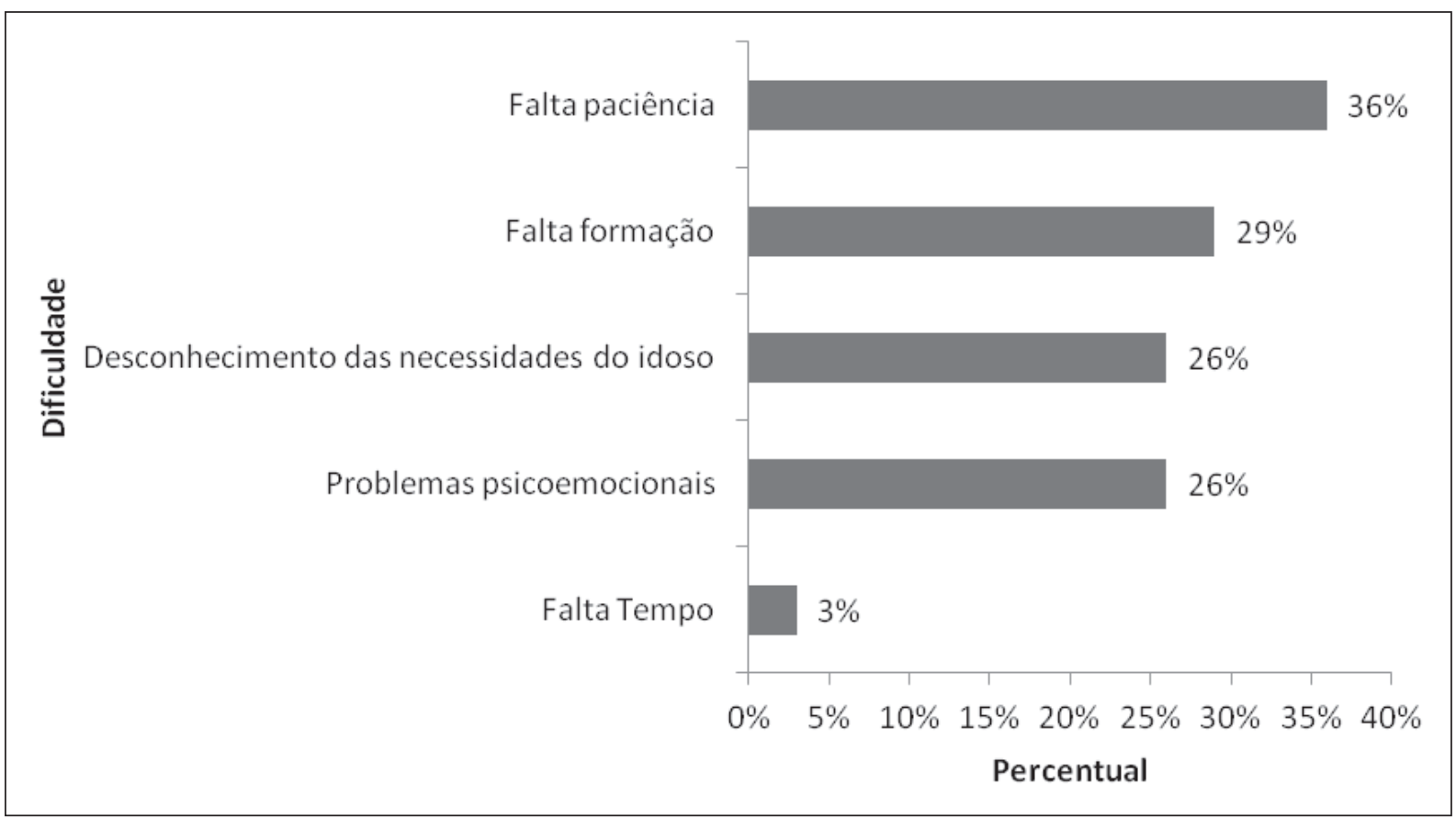

Figura 1 - Principais dificuldades enfrentadas pelos cuidadores, ESF Seringal. Ananindeua, PA, 2010-2011.

\section{DISCUSSÃO}

O envelhecimento é um processo biológico cujas alterações determinam mudanças estruturais no corpo e, em decorrência desta, modificam suas funções. O envelhecer, contudo, é inerente a todo ser vivo e no homem este processo assume dimensões biológicas, sociais e psicológicas.

A idade dos entrevistados esteve na faixa dos 50 anos. Esse predomínio permite relacionar que nessa fase da vida adulta o ser humano requer uma maior atenção psicosociobiológica, o que nem sempre é uma situação fácil para os cuidadores, que poderão ter complicações com o cuidado de si.

Muitos estudos demonstram que cuidar não é uma tarefa fácil, exige uma mudança radical na vida de quem cuida e também demanda a execução de tarefas complexas, delicadas e sofridas. Em muitos casos, o cuidador é também uma pessoa frágil, já em idade de envelhecimento ou em vias de ficar doente.?

O cuidador sem suporte pode ser o futuro idoso cuidado, pois a experiência de estar incapacitado ou de se tornar um cuidador gera uma mudança radical na vida das pessoas. Essas mudanças podem vir seguidas de sofrimento, decorrente de doença crônica ou de alguma dependência física. ${ }^{10}$

O processo de envelhecimento é uma extensão lógica dos processos fisiológicos do crescimento, desenvolvimento e interação com os fatores ambientais, resultando em perda na capacidade de reserva e redundância que reduz a capacidade de se adaptar rápida e eficientemente ao meio. ${ }^{11}$ 
$\mathrm{Na}$ sociedade, apesar das mudanças recentes, a figura masculina está geralmente associada ao trabalho fora do lar, do homem saindo para trabalhar e prover o sustento de sua família; e da mulher, ficando em casa como responsável pelo lar e pelo cuidado dos membros da família. Embora venham ocorrendo grandes mudanças neste contexto atualmente, esse imaginário ainda exerce grande influência e pode ajudar a explicar os dados obtidos com esta pesquisa, na qual o gênero de cuidadores predominante foi o feminino.

Este dado é reforçado pela pesquisa realizada por Oliveira e colaboradores, ${ }^{12}$ segundo a qual $70,3 \%$ dos cuidadores entrevistados eram mulheres, sendo que mesmo com a inserção da mulher no mercado de trabalho, como demonstrado na pesquisa, ela ainda é a principal responsável pelo cuidado de idosos dependentes.

Este fato reflete as relações de gênero que cada sujeito exerce frente a seus papéis na família, em que o princípio tradicionalista da divisão sexual do trabalho dita a predominância do padrão de domesticidade, que confere desvantagens às mulheres na vida social em geral. Esse padrão, todavia, possui variações internas, e é mediado por aspectos culturais e socioeconômicos que lhes determinam configurações particulares de acordo com determinadas variáveis socioeconômicas e/ou com os valores mais gerais que orientam as ações. ${ }^{13}$

Historicamente, a mulher sempre foi responsável pelo cuidado, seja da casa ou dos filhos, ou mesmo dos membros familiares adoecidos, enquanto o papel do homem era de trabalhar fora para garantir o provimento financeiro da família. Apesar de todas as mudanças sociais e na composição familiar, e dos novos papéis assumidos pela mulher, destacando-se sua maior participação no mercado de trabalho, ainda se espera que a mulher assuma as funções do cuidado em geral. ${ }^{14}$

É comum que as mulheres assumam os cuidados mesmo quando trabalham fora, o que acaba repercutindo em mais limitações de tempo livre e implicações no cuidado de si. Geralmente, os homens participam do cuidado de forma secundária, por meio de ajuda material ou em tarefas externas, como o transporte do idoso, compra de remédios ou mesmo no pagamento de contas, como encontrado em alguns diálogos durante a realização deste estudo. ${ }^{14}$

Com relação ao grau de escolaridade, observou-se que a maioria dos cuidadores possuía ensino médio completo, mas entendese que esta característica inerente aos mesmos não se diferenciou quando correlacionados com as principais dificuldades encontradas no ato de cuidar. Além disso, a pesquisa revelou que cuidadores com escolaridade alta eram afetados pelos mesmos problemas de cuidadores com nível de escolaridade mais baixa.

Estudos demonstram, entretanto, que a escolaridade pode influenciar na qualidade da assistência ao idoso, uma vez que o cuidador precisa seguir dietas, ler bulas de remédios, entender dosagens e via de administração para melhor prover o cuidado, além da valorização e conscientização sobre a importância na prevenção de doenças na terceira idade..$^{15}$

É importante conhecer a escolaridade dos cuidadores, pois são eles que colhem as informações junto aos idosos e as repassa a equipe de saúde. A educação em saúde neste ponto está muito ligada à capacidade de aprendizagem das pessoas, revelando-se como um laço de ligação entre seu cuidado e o cuidado ao outro. Assim, a escolaridade do sujeito, seja um cuidador primário ou mesmo secundário, influencia diretamente na assistência prestada. ${ }^{16}$

O nível de escolaridade também influencia nos sentimentos dos cuidadores em relação ao cuidado do outro, sendo que a pouca aprendizagem dificultaria a compreensão do que acontece com o idoso, das dificuldades encontradas e enfrentadas ao cuidar. Este fato é relevante, uma vez que os sentimentos dos cuidadores são consequência da interação complexa de fatores como convivência, medo 
e empatia, indo muito além das informações patológicas repassadas pela equipe de saúde durante as consultas ambulatoriais dos idosos. ${ }^{10}$

Quanto à situação conjugal, observou-se que a maioria dos cuidadores possuía relações estáveis, dado que sinaliza que, além da tarefa de cuidar do idoso, estes possuem outras tarefas e responsabilidades, como cuidar da casa, preparar as refeições da família e atender às solicitudes dos filhos, o que pode resultar em sobrecarga de atividades. Isto pode ser observado na pesquisa de Somerheld \& Neri, ${ }^{17}$ em que os cuidadores referiram que cuidar era um trabalho árduo, gerador de sobrecarga e resultava em cansaço, insônia e problemas de saúde.

Os dados encontrados refletem que a situação conjugal mostra ser um apoio importante para os cuidadores de idosos quando o cuidado é dividido entre os cônjuges. Entretanto, quando não compartilhado, pode se tornar um fator agravante à saúde do cuidador, pois essa relação implica diretamente a realização do cuidado de si, devido à falta de tempo direcionado a este ato.

Em muitos casos, devido às dificuldades financeiras enfrentadas durante o cuidar, o cuidador precisa reduzir o tempo dedicado ao trabalho externo ou mesmo a busca de um provento pessoal. A preocupação com o cuidado do idoso passa a ser foco do casal, que muitas vezes não pode contar com serviços de cuidado domiciliar especializados, nem realizar reformas para diminuir a sobrecarga. ${ }^{9}$ Dessa forma, os cônjuges acabam por experimentar limitações em manter uma vida social ativa, reduzindo os encontros com parentes e amigos. No longo prazo, vivenciam a redução de visitas em seus lares, dada a dedicação, muitas vezes, exclusiva ao idoso. ${ }^{17}$

Constatou-se ainda que a maioria dos cuidadores não é recompensada financeiramente pelo cuidado prestado; dessa forma, precisa estabelecer trabalhos secundários ao cuidar, a fim de prover o sustento de sua família. A remuneração mensal ficou em média de dois a três salários mínimos, tendo o cuidador um vínculo informal com o ser cuidado.

Com base nesses dados, é possível afirmar que a maior parte dos cuidadores vive com renda própria, e a minoria com a renda familiar proveniente das pensões e benefícios dos idosos. Esse fato mostra que o universo dos cuidadores de idosos em relação às correntes financeiras estabelecidas durante o ato de cuidar apresentase como um universo a ser desvendado, que muitas vezes vai de encontro ao que se pensa o senso comum que vincula o cuidador em uma relação harmônica com o cuidar, revelando novos dilemas e ações a serem esclarecidos e organizados pela equipe de saúde. ${ }^{6}$

A sobrecarga financeira é fator gerador de estresse e desgaste físico, não só do cuidador, mas de toda a família, pois o familiar cuidador geralmente tem problemas com o emprego, ao abandonar ou reduzir a jornada de trabalho, saindo mais cedo ou mesmo largando o emprego para se dedicar ao cuidado do idoso. ${ }^{13}$

Oimpacto nas relações familiares causado pelo cuidado ao idoso e suas consequências mostrase muito forte, como decorrência das alterações inevitáveis que envolvem afeto, finanças, relações de poder e outras variáveis. Desenvolve-se então um processo de reorganização familiar, quando alguém deixa de executar tarefas pessoais, domésticas e sociais em prol do outro. ${ }^{18,19}$

Quanto ao item convivência, prevaleceu entre os entrevistados o vínculo afetivo com o cuidar há mais de um ano, sendo em sua quase totalidade exercida pelos próprios filhos dos idosos. Esse fato demonstra que o apoio dos familiares no exercício do ato de cuidar é crucial para os idosos enfrentarem as enfermidades. 
Neste sentido, os profissionais de saúde, enquanto colaboradores dos familiares cuidadores, devem exercer papel ativo neste cenário, buscando sempre inserir a família no cuidado e, com isso, diminuir as sobrecargas individuais, evitando transformar o cuidador em uma pessoa que futuramente possa se tornar um ser que venha necessitar de cuidado. ${ }^{5}$

O cuidado domiciliar proporciona uma série de pontos positivos para todos os envolvidos no tratamento: para o idoso, a proximidade do ambiente familiar acelera o processo de recuperação e evita o deslocamento de familiares para o hospital; para a família, o fato de poder estar constantemente com o idoso, poder tratá-lo com mais atenção, ter a oportunidade de fazer o que acredita ser melhor, mantém e reforça laços afetivos. Além disso, há maior humanização no cuidado do paciente pelos cuidadores. ${ }^{2,6}$

A informação sobre o idoso, neste caso, converge no ponto precursor entre a manutenção e o abalo do processo saúde e doença, em que quanto mais positivo o sujeito de cuidados reconhece o ser cuidado, mais serão as chances de promoção de cuidados e prevenção de agravos ao idoso, de forma a facilitar a reabilitação, inclusão, autonomia e independência., ${ }^{2,3}$

A maior parte dos entrevistados referenciou que as principais dificuldades encontradas durante os cuidados com os idosos foram a falta de formação para cuidadores e a falta de paciência. Relataram realizar o trabalho de forma experimental, semelhante à pesquisa de Caldeira \& Ribeiro, ${ }^{20}$ que ressaltou a complexidade da função e o despreparo dos cuidadores revelados por meio de incertezas e de imprevisibilidades de cuidados, abalando a moral e a crença do cuidador, pois este não sabe se proporciona um cuidado digno ao idoso.

No entanto, os dados que evidenciam a impaciência se contrapõem aos números observados por Gratão, ${ }^{21}$ tendo em vista que o estresse durante as atividades com o idoso e a irritação devido ao convívio com ele não foram citadas como atividades que dificultam, tampouco causam sobrecarga aos cuidadores. Esse zelo no tratamento com os idosos pode ser explicado, pois $53 \%$ dos cuidadores que participaram da pesquisa realizada por Gonçalves et al. $^{22}$ afirmaram que as tarefas desempenhadas nesse ofício os dignificam como pessoa e, para 52\%, trata-se de uma obrigação ética.

Os dados encontrados, mesmos que comparados com outros estudos, se limitam a uma realidade local do grupo estudado, pois se trata de um recorte que reflete características específicas dessa população, necessitando assim de mais pesquisas para fomentar um maior levantamento das particularidades e dificuldades enfrentadas pelos cuidadores.

\section{CONCLUSÃO}

A maioria dos sujeitos cuidadores de idosos era formada por mulheres casadas, filhas dos idosos e que não dispunham de nenhuma renda para efetivação do exercício de cuidar. Consideraram o idoso sob seu cuidado como saudável, tendo suas principais dificuldades para o ato de cuidar a falta de paciência e a falta de formação para exercer a função.

Nesse contexto, é de suma importância que se conheça o universo dos cuidadores, assim como seu relacionamento com o idoso, para que a equipe da Estratégia de Saúde da Família possa traçar estratégias de saúde, segundo as necessidades e a acessibilidade dos indivíduos. Quando o idoso recebe as orientações médicas, são os cuidadores domiciliares que irão dar continuidade ao tratamento necessário para a convalescência do sujeito em seu domicílio. 


\section{REFERÊNCIAS}

1. Garbin CAS, Sumida DH, Moimaz SAS, Prado RL, Silva MM. O envelhecimento na perspectiva do cuidador de idosos. Ciênc Saúde Coletiva 2010;15(6):2941-48

2. Abreu CB, Ribeiro MI, Pires NR. Cuidando de quem já cuidou: o livro do cuidador. São Paulo: Atheneu; 2009.

3. Farias RG, Santos SMA. Influência dos determinantes do envelhecimento ativo entre idosos mais idosos. Texto Contexto - Enferm 2012;21(1):167-76.

4. Gaioli CCLO, Furegato ARF, Santos JLF. Perfil de cuidadores de idosos com doença de Alzheimer associado à resiliência. Texto Contexto - Enferm 2012;21(1):150-57.

5. Araujo JS, Silva SED, Santana ME, Conceição VM, Vasconcelos EV. O processo do cuidar/cuidado nas representações sociais de cuidadores de pacientes sequelados por acidente vascular cerebral. Rev Enferm em Foco 2011;2(4):235-38.

6. Araujo JS, Silva SED, Santana ME, Vasconcelos EV, Conceição VM. Yes, i know what is the spill. The social representation of caregivers about the stroke. Rev Pesquisa: Cuidado é Fundamental 2012;4(1):2849-59.

7. Araújo JS, Silva SED, Santana ME, Vasconcelos EV, Conceição VM. A obrigação de (des) cuidar: representações sociais sobre o cuidado à sequelados de acidente vascular cerebral por seus cuidadores. REME - Rev Min Enferm 2012;16(1):98-105.

8. Sampaio AMO, Rodrigues FN, Pereira VG, Rodrigues SM, Dias CA. Cuidadores de idosos: percepção sobre o envelhecimento e sua influência sobre o ato de cuidar. Estud Pesqui Psicol 2011;11(2):590-613.

9. Papaleo Neto M. Gerontologia: a velhice e o envelhecimento em visão globalizada. São Paulo: Atheneu; 2005. 524 p.

10. Andrade LM, Costa MFM, Caetano JÁ, Soares E, Beserra EP. A problemática do cuidador familiar do portador de acidente vascular cerebral. Rev Esc Enferm USP 2009;43(1):37-43.

11. Graças EM, Santos GF. Metodologia do cuidar em enfermagem na abordagem fenomenológico. Rev Esc Enferm USP 2009;43(1):200-7.

12. Oliveira SK, Landgraf Junior FJ, Dellaroza MSG, Yamada KN, Trelha CS, Cabrera MAS. Perfil dos cuidadores de idosos atendidos pelo Projeto Assistência Interdisciplinar a Idosos em Nivel Primário - PAINP, Londrina-PR. Ciência, Cuidado e Saúde 2006;5(2):184-92.

13. Cavalcante LF. Gênero, família e trabalho no Brasil. Rio de Janeiro: FGV; 2010.

14. Freitas ICC, Paula KCC, Soares JL, Parente ACM. Convivendo com o portador de Alzheimer: perspectivas do familiar cuidador. Rev Bras Enferm 2008;61(4):508-13.

15. Nakatani AYK, Souto CCS, Paulette LM, Melo TS, Souza MM. Perfil dos cuidadores informais de idosos com déficit de autocuidado atendidos pelo Programa de Saúde da Família. Rev Eletrônica de Enferm [periódico na Internet]. 2003 [Acesso em 25 nov 2011];5(1):[aproximadamente Xp.]. Disponível em: http://www.revistas.ufg.br/.

16. Mayor MS, Ribeiro O, Paul C. Satisfaction in dementia and stroke caregivers: a comparative study. Rev Latino-Am Enfermagem 2009;17(5):620-4.

17. Sommerhalder C, Neri AL. Avaliação subjetiva da tarefa de cuidar: ônus e benefícios percebidos por cuidadoras familiares de idosos de alta dependência. In: Neri AL, organizadora. Cuidados ao cuidador: questões psicossociais. Campinas: Átomos Alinea; 2001. p. $91-132$.

18. Rocha MPF, Vieira MA, Sena RR. Desvelando o cotidiano dos cuidadores informais de idosos. Rev Bras Enferm 2008;61(6):238-47

19. Azevedo GR, Santos VLCG. Cuida-dor (D) eficiente: as representações sociais de familiares acerca do processo de cuidar. Rev Latino-am Enfermagem 2006;14(5):770-80.

20. Caldeira APS, Ribeiro RCHM. O enfrentamento do cuidador do idoso com Alzheimer. Arq Ciênc Saúde 2004;11(2):100-4.

21. Gratão ACM. Sobrecagra vivenciada por cuidadores de idosos na comunidade [tese de doutorado]. Ribeirão Preto: Escola de Enfermagem de Ribeirão Preto da Universidade de São Paulo; 2010.

22. Gonçalves LHT, Alvarez AM, Sena ELS, Santana LWS, Vicente FR. Perfil da família cuidadora de idoso doente/fragilizado do contexto sociocultural de Florianópolis, SC. Texto Contexto Enferm 2006;15(4):570-7. 